\title{
Placenta Accreta: Management by Peridural Anesthesia
}

\author{
Francesco Tarantino ${ }^{1}$ and Giuseppe Cali $^{1}$ \\ ${ }^{1}$ Villa Sofia Cervello United Hospitals
}

June 18, 2020

Placenta Accreta: Management by Peridural Anesthesia

Francesco Tarantino, ${ }^{\mathrm{a}}$ Giuseppe Calì ${ }^{\mathrm{b}}$

a Anesthesia and Intensive Care Unit 2, Villa Sofia-Cervello Hospital, Palermo

${ }^{\mathrm{b}}$ Gynecology and Obstetric Unit, Villa Sofia-Cervello Hospital, Palermo, Italy. Person in charge for the Perinatal Medicine and Assistance to the Birth Unit at the ARNAS CIVICO of Palermo, Department of Maternal and Nascent Life Health

Correspondence : Francesco Tarantino, Anesthesia and Intensive Care Unit 2, Villa Sofia-Cervello Hospital, Viale Strasburgo, 233, 90146 Palermo, Italy. Phone: +393207032427. Email: tarantino.f@virgilio.it

Running title: Peridural Anesthesia in Placenta Accreta surgery

\section{Introduction}

As a consequence of the extensive use of cesarean sections, the incidence of placenta accreta spectrum (PAS) disorders, characterized by abnormal invasion of trophoblastic tissue through the myometrium and uterine serosa, has been growing considerably ${ }^{1}$. This condition exposes mothers to the risk of massive bleeding (average blood loss $\sim 2-5 \mathrm{~L}^{2}$ ) and death. The maternal mortality rate in its most severe form (placenta percreta) is reportedly $7 \%^{3}$.

The most recent guidelines ${ }^{4}$ suggest producing in-hospital protocols that are shared among all involved professionals and establishing a step-by-step plan, from ultrasound-based detection of placenta previa to post-surgery management.

\section{Our internal protocol}

The present article intends to share the internal protocol developed in our center, Villa Sofia-Cervello Hospital, Palermo, Italy (Figure 1).

Our hospital meets the criteria outlined by the American College of Obstetricians and Gynecologists (ACOG) and the Society for Maternal-Fetal Medicine to provide the most appropriate level of maternal care required for the management of PAS disorders ${ }^{4}$. This article focuses on the anesthesiologic management of the procedure, while the surgical management is not detailed herein. 


\section{From diagnosis to surgery}

Table 1 describes the steps we follow from diagnosis to surgery. In particular, early diagnosis allows for a multidisciplinary team, careful tailoring of the management plan, and adequate preparation of the elective cesarean section and subsequent hysterectomy.

\section{Anesthesiology management}

To date, there is no consensus on the best type of anesthesia in cases of complete placenta previa with suspected accretism. However, historically, the most frequently used technique is general anesthesia ${ }^{8}$, which is generally preferred because it reduces the mother's anxiety, allows the anesthetist to concentrate on the hemorrhage and cardiovascular stability, and avoids the risk of hemodynamic instability due to an urgent shift (due to hemorrhage) from locoregional to general anesthesia.

Our internal protocol, which indicates general anesthesia in emergency regimens, provides the use of peridural anesthesia not only during birth but also during the hysterectomy, considering the shift to general anesthesia in case of emergency (bleeding or hemodynamic instability). From our point of view, several beneficial effects may be achieved with peridural analgesia, such as:

- reduced risk of Mendelson syndrome;

- better post-surgery pain control;

- no risk of awareness;

- no risk for the fetus being exposed to the effect of general anesthetics;

- participation of the mother in the birth of her baby;

- continuous monitoring of the patient's level of consciousness, which indicates the level of brain perfusion and, thus, of hemodynamic status;

- abolition of the side effects of general anesthetics, such as the reduction of the uterine tone and platelet functionality.

Comparing the current state of the art with that of the past, the pivotal role of the interventional radiologist is apparent in reducing the vascular inflow to the operating field and, in case of hemorrhage during the hysterectomy, to stop the bleeding ${ }^{6}$. Therefore, it is now possible to use locoregional anesthesia in obstetrics to have good outcomes, such as reduced bleeding, reduced use of blood products in the major obstetric hemorrhages, and better management in post-surgery pain therapy. Finally, we chose peridural anesthesia over combined spino-epidural technique because the peridural catheter is placed in the obstetrics operatory room 60 minutes (radiological time) before the skin incision in the interventional radiology room. Therefore, the maximum effect of spinal anesthesia would begin to decrease. In our experience, the peridural anesthesia has a reduced hemodynamic effect compared with the spino-epidural technique.

During the entire procedure, two anesthetists from the dedicated group of obstetrics are present.

\section{Obstetric operatory room}

The patient was prepared in the obstetrics operatory room after fasting for at least 8 hours. During this phase, the anesthesiologists explained the procedure step by step to the patient. Two large peripheral veins are cannulated with $16 \mathrm{G}$ or $18 \mathrm{G}$ cannulas. Antibiotic prophylaxis and $7 \mathrm{ml} / \mathrm{kg}$ saline (to replace fluids lost through fasting) are administered. While maintaining the absolute sterility of the operating field, the skin is disinfected. Cutaneous wheal is formed using $4-6 \mathrm{~mL}$ of $2 \%$ lidocaine. The peridural space is identified, generally at the L2-L3 level, using the loss-of-resistance technique. The peridural catheter (16 or $18 \mathrm{G}$ ) is placed on the patient in a sitting or lateral position on the operating bed, while electrocardiogram (ECG), oxygen saturation, and noninvasive blood pressure (NIBP) are monitored. Once the peridural space is identified, the catheter is introduced in the cranial direction approximately $3 \mathrm{~cm}$ beyond the bevel of the Tuohy needle. The aspiration test (to rule out the presence of blood or liquor) and dose test are performed with $2 \mathrm{ml}$ of $2 \%$ lidocaine. Afterwards, the catheter is attached to the skin. The operating room cart is 
prepared (Supporting Information 2) by nurses supervised by the anesthetist. Finally, the patient and the cart are moved to the interventional radiology room.

\section{Interventional radiology room}

Time 1

The patient is placed on an X-ray transparent operating bed and warmed up with a thermal mattress. The equipment and devices to be used in the radiological procedure are already in this room (digital angiograph, $\mathrm{C}$ arch), as well as the respirator and the dedicated space for the neonate. Colloids $(10 \mathrm{~mL} / \mathrm{kg})$ are infused intravenously and vital parameters are monitored. Subsequently, two boluses of $14-18 \mathrm{~mL}$ of $0.75 \%$ ropivacaine (105-135 mg) and fentanyl $50 \gamma$ are administered 7-8 minutes apart to avoid hypotensive episodes.Radial artery cannulation is performed to monitor the arterial pressure during the whole surgical intervention and for serial arterial blood gas analysis before and after the surgery (to monitor hemoglobin levels and metabolic status). A central venous catheter is also placed in case of difficult access of other peripheral veins. A wedgeis placed under the right hip of the patient to avoid compression of the uterus on the vena cava. While waiting for the maximum effect of anesthetics, invasive blood pressure, oxygen saturation, electrocardiogram, and body temperature are monitored, and a pre-surgery arterial blood gas analysis is performed. After approximately 15 minutes, the Pin Prick test is done to evaluate the anesthetic plan of the central block in terms of depth, width, and symmetry. Hollmen and Bromage scales are useful in the assessment of the dermatomal level achieved. When the anesthetic plan reaches the T4-T5 dermatomal level and the Hollmen scale is 3, urinary catheterization may be performed, and the radiological-vascular time starts with the placement of endovascular catheters in the hypogastric arteries .

Time 2

Once the radiological examination is completed, cesarean section is performed, generally using a supraumbilical-pubic incision. The patient is then awake, has no pain, and participates in the birth of her child. After extraction, the premature fetus is handed to the neonatology team. The mother is given uterotonic agents intravenously to trigger uterine contraction, facilitate the possible placental stage, and reduce further blood loss.

Time 3

If the placenta is not delivered, morbid placenta adhesion is confirmed. Therefore, the obstetric team performs the riskiest phase of the surgery: hysterectomy . In this phase, in order to reduce the mother's anxiety, a mild sedation may be administered with i.v. benzodiazepine (midazolam) and, in the absence of apparent hemorrhage signs, after several minutes, a $1.5 \mathrm{mg} / \mathrm{kg}$ bolus of i.v. propofol may be slowly administered (over approximately 90 seconds) so that the patient can breathe spontaneously with a Ventimask $\left(40 \%, \mathrm{O}_{2}\right.$ flow 8 $\mathrm{L} / \mathrm{min})$. Subsequently, a continuous infusion of propofol may be given $(1.5-3 \mathrm{mg} / \mathrm{kg} / \mathrm{h})$. Sedation has some advantages:

- the patient does not feel possible visceral pain due to traction and adherence to previous surgeries;

- it strengthens the status of controlled hypotension, further reducing intraoperative bleeding; and

- it allows for an easier shift to general anesthesia, if necessary.

Time 4

The last part of the procedure is proper evaluation of the possible hemorrhages . For the management of hemorrhagic emergency, in accordance with the current guidelines of the Italian Ministry of Health ${ }^{10}$, an operative protocol was drawn up and is followed (see Supporting Information 3). Finally, the possible referral to ICU is considered, taking into account the amount of bleeding, the type of anesthesia, the length of surgery, the hemodynamic stability, and the level of consciousness. Otherwise, after suturing the abdominal belt, sedation is suspended, with awakening of the patient in a very short time. 


\section{Post-surgery monitoring}

After surgery, patients remain under observation in the recovery room in the obstetrics and gynecology unit, where they are monitored and warmed up, assisted by an anesthetist, a midwife, and a nurse. All parameters are registered in the medical record. After 4 hours, if the vital parameters are within the normal range, the Aldrete score is 9-10, and the numerical rating scale (NRS) is 2, the patient is moved to the ward, where the monitoring can be continued. The blood count is controlled every 4 hours in the first 12 hours, and subsequently every 8-12 hours, according to hemoglobin and hematocrit levels. For the first 6 hours after surgery, continuous pressure monitoring is performed, in addition to pulse oximetry, urine output measurement with fluid balance, thromboelastography, serum electrolytes test, and kidney function tests.

\section{Post-surgery pain control}

For the 24/48-hour peridural pain management, we follow the protocol reported in Supporting Information 4. Briefly, pain management is administered via the pump for programmed intermittent epidural bolus (PIEB), through which boluses of $8-12 \mathrm{ml}$ of $0.1 \%$ ropivacaine (hourly) and acetaminophen (at established times) are administered. The peridural catheter is removed on the third day, 12 hours after the last low-molecularweight heparin administration, and upon assessment of possible alterations in coagulation status.

\section{Conclusions}

The efficiency of this protocol lead to our becoming a reference center for the management of placenta accreta beyond the borders of our region. Logistically, the process is made easier because all the operative units involved are located in the same building.

In these high-risk patients, some elements were very important:

- early prenatal diagnosis;

- referral of patients in qualified hospitals with expertise in the management of such conditions;

- involvement of a multidisciplinary team;

- right surgical timing;

- proper peri-surgery management.

Using the protocol described, we treated more than 20 patients, some of them with the most severe forms of PAS disorders (placenta increta and percreta). We obtained positive results in terms of bleeding, packed red blood cells used, postoperative sequelae, and UTI admissions. No patients died. Most patients had a previous cesarean section.

Few procedures were carried out in an emergency regimen, where general anesthesia was used. Consistent with literature ${ }^{8}$, they required the greatest amount of blood products. However, based on our experience for both elective and emergency surgeries, fibrinogen administration considerably reduced the need for PRBC. Among those treated with an elective regimen, blood loss never exceeded $1800 \mathrm{~mL}$.

\section{Acknowledgements}

We acknowledge CSL Behring, Italy, which funded the online open publication, publishing support, and journal styling services, that were provided by SEEd Medical Publishers. 


\section{Disclosure of Interests}

Dr. Tarantino reports non-financial support from CSL the conduct of the study; personal fees from Boehringer, outside the submitted work. Dr. Calì reports non-financial support from CSL Behring, during the conduct of the study.

\section{Contribution to Authorship}

FC: Conceptualization, preparation, manuscript review, and editing. GC: Supervision, manuscript review.

\section{Funding}

Publishing support, journal styling services, and open access publication of this article were funded by CSL Behring, Italy. The sponsor had no role in the collection, analysis, and interpretation of data or writing of the final statistical report.

\section{References}

1. Morlando M, Sarno L, Napolitano R, Capone A, Tessitore G, Maruotti GM, et al. Placenta accreta: incidence and risk factors in an area with a particularly high rate of cesarean section. Acta Obstet Gynecol Scand. 2013 Apr;92(4):457-60.

2. DeSimone RA, Leung WK, Schwartz J. Transfusion Medicine in a Multidisciplinary Approach to Morbidly Adherent Placenta: Preparing for and Preventing the Worst. Transfus Med Rev. 2018;32(4):244-8.

3. O'Brien JM, Barton JR, Donaldson ES. The management of placenta percreta: conservative and operative strategies. Am J Obstet Gynecol. 1996 Dec;175(6):1632-8.

4. Society of Gynecologic Oncology, American College of Obstetricians and Gynecologists and the Society for Maternal-Fetal Medicine, Cahill AG, Beigi R, Heine RP, Silver RM, et al. Placenta Accreta Spectrum. Am J Obstet Gynecol. 2018;219(6):B2-16.

5. Jauniaux E, Chantraine F, Silver RM, Langhoff-Roos J, FIGO Placenta Accreta Diagnosis and Management Expert Consensus Panel. FIGO consensus guidelines on placenta accreta spectrum disorders: Epidemiology. Int J Gynaecol Obstet. 2018 Mar;140(3):265-73.

6. Di Mascio D, Calì G, D'antonio F. Updates on the management of placenta accreta spectrum. Minerva Ginecol. 2019 Apr;71(2):113-20.

7. Shamshirsaz AA, Fox KA, Erfani H, Clark SL, Salmanian B, Baker BW, et al. Multidisciplinary team learning in the management of the morbidly adherent placenta: outcome improvements over time. Am J Obstet Gynecol. 2017;216(6):612.e1-612.e5.

8. Allen L, Jauniaux E, Hobson S, Papillon-Smith J, Belfort MA, FIGO Placenta Accreta Diagnosis and Management Expert Consensus Panel. FIGO consensus guidelines on placenta accreta spectrum disorders: Nonconservative surgical management. Int J Gynaecol Obstet. 2018 Mar;140(3):281-90.

9. ASA Physical Status Classification System [Internet]. [cited 2020 May 17]. Available from: https://www.asahq.org/standards-and-guidelines/asa-physical-status-classification-system

10. Ministero della Salute. Parto, Linea guida nazionale per la prevenzione e il trattamento dell'emorragia post partum [Internet]. [cited 2020 May 17]. Available from:http://www.salute.gov.it/portale/news/p3_2_1_1_1.jsp?lingua=italiano\&̈menu=notizie\& $p=$ dalministero\&id $=2740$ 


\section{Tables}

Table 1. Management steps from the diagnostic suspicion to the surgery

Steps Description

Ultrasonography

Magnetic resonance imaging

\section{Involvement of a multidisciplinary team and planning}

\section{Preoperative anesthetic examination and informed consent}

Multidisciplinary discussion
Routine ultrasonography, ideally performed before the third trimester $(22-32 \text { weeks })^{5}$, allows identification of the first signs of PAS disorders; after an obstetric-gynecological consultancy, a re-evaluation from two experienced sonographers is required

Magnetic resonance imaging is indicated to confirm the diagnosis and better delineate the degree of trophoblastic invasion and its correlation with the anastomotic system of vessels in that area $^{6}$

After diagnosis confirmation, the delivery should be planned, with the involvement of a multidisciplinary team, including a gynecologist, anesthesiologist, neonatologist, urologist, and interventional radiologist, in addition to obstetricians and nurses ${ }^{7}$, that will follow the patients up to the end of post-surgery monitoring and manage pain control. Elective cesarean section is recommended at $36-37$ weeks $^{8}$ in asymptomatic women, in case of suspicion or diagnosis of PAS disorder, with respiratory distress syndrome prophylaxis. However, the exact timing is tailored for each pregnant woman by the gynecologist, who evaluates the risks for the mother and the gestational age of the fetus During the preoperative anesthetic examination, the anesthesiologist first meets the patient and assesses general condition, airways, venous accesses, vertebral column status, and hemodynamic stability (Supporting Information 1); finally, the patient is given the score according to the American Society of Anesthesiologists (ASA) classification $^{9}$. In this first visit, the anesthesiologist establishes a relationship based on trust, explains the phases of surgery in detail, and collects the informed consent, which includes all the possible interventions and complications (hysterectomy, lesions to the surrounding organs, need for blood product transfusions, admission in the Intensive Care Unit [ICU])

A multidisciplinary discussion takes place to coordinate the health professionals involved in the management of the patient. 


\begin{tabular}{ll}
\hline Steps & Description \\
\hline Transfusion service and ICU alerted & About 3 days before surgery, the transfusion \\
service should also be alerted: 4 units of packed \\
red blood cells (PRBC) should be stocked in the \\
blood bank of the operating room the day of \\
surgery and further 4 units should be stocked on \\
the hospital transfusion center. In addition, $6 \mathrm{~g}$ of \\
fibrinogen and tranexamic acid should be \\
available in the operating room. ICU is alerted to \\
reserve a bed in case of need
\end{tabular}

\section{Figure Caption List}

Figure 1. Summary of the internal protocol for the management of placenta accreta spectrum disorders in the Villa Sofia-Cervello Hospital, Palermo, Italy

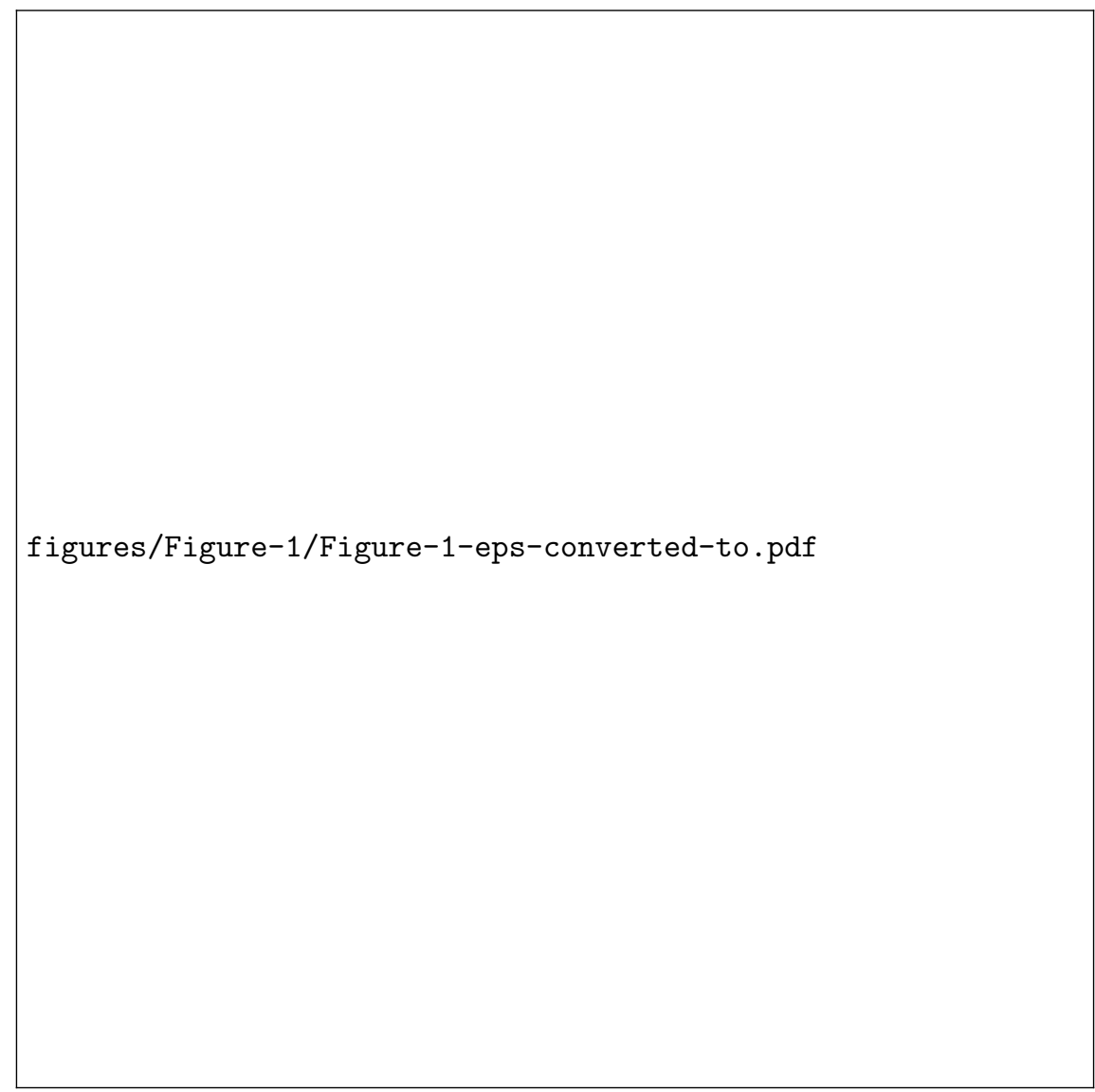

